\title{
Chapter 8 \\ X-Ray, Radio and SEP Observations of Relativistic Gamma-Ray Events
}

\author{
Karl-Ludwig Klein, Kostas Tziotziou, Pietro Zucca, Eino Valtonen, \\ Nicole Vilmer, Olga E. Malandraki, Clarisse Hamadache, Bernd Heber, \\ and Jürgen Kiener
}

\begin{abstract}
The rather frequent occurrence, and sometimes long duration, of $\gamma$ ray events at photon energies above $100 \mathrm{MeV}$ challenges our understanding of particle acceleration processes at the Sun. The emission is ascribed to pion-decay photons due to protons with energies above $300 \mathrm{MeV}$. We study the X-ray and radio emissions and the solar energetic particles (SEPs) in space for a set of 25 Fermi $\gamma$-ray events. They are accompanied by strong SEP events, including, in most cases where the parent activity is well-connected, protons above $300 \mathrm{MeV}$. Signatures of energetic electron acceleration in the corona accompany the impulsive and early post-impulsive $\gamma$-ray emission. $\gamma$-ray emission lasting several hours accompanies in general the decay phase of long-lasting soft X-ray bursts and decametric-tokilometric type II bursts. We discuss the impact of these results on the origin of the $\gamma$-ray events.
\end{abstract}

\author{
K.-L. Klein $(\bowtie) \bullet N$. Vilmer $\bullet$ P. Zucca \\ LESIA-Observatoire de Paris, CNRS, 92190 Meudon, France
}

PSL Research University, Universités P \& M. Curie, Paris-Diderot, Meudon, France

e-mail: ludwig.klein@obspm.fr; pietro.zucca@obspm.fr; nicole.vilmer@obspm.fr

K. Tziotziou • O.E. Malandraki

National Observatory of Athens, IAASARS, Athens, Greece

e-mail: kostas@noa.gr; omaland@astro.noa.gr

E. Valtonen

Department of Physics and Astronomy, Space Research Laboratory, University of Turku, Turku, Finland

e-mail: eino.valtonen@utu.fi

C. Hamadache • J. Kiener

CSNSM, IN2P3-CNRS, Univ. Paris-Sud, 91405 Orsay Cedex, France

e-mail: clarisse.hamadache@csnsm.in2p3.fr; Jurgen.kiener@csnsm.in2p3.fr

B. Heber

Christian-Albrechts-Universität zu Kiel, Kiel, Germany

e-mail: heber@physik.uni-kiel.de

(C) The Author(s) 2018

O.E. Malandraki, N.B. Crosby (eds.), Solar Particle Radiation Storms Forecasting

and Analysis, The HESPERIA HORIZON 2020 Project and Beyond, Astrophysics

and Space Science Library 444, DOI 10.1007/978-3-319-60051-2_8 


\subsection{Introduction}

The advent of the Fermi mission showed that the Sun is an occasional, but unexpectedly frequent, emitter of $\gamma$-ray photons above $100 \mathrm{MeV}$. These are understood to be produced by pion decays in nuclear interactions involving protons or He-nuclei at energies above $300 \mathrm{MeV} /$ nucleon. One did not expect that the Sun was able to accelerate relativistic protons and nuclei even in seemingly modest flares. These particles are rarely detected in space ( $<1$ event per year). Furthermore, the duration, several hours, of some $\gamma$-ray events is much longer than that of hard X-ray signatures of electron acceleration in the impulsive flare phase.

The question of how the $\gamma$-ray emission is related to other signatures of particle acceleration and energy release in the corona is crucial to understanding the origin of the high-energy protons. It might also be expected that such high-energy populations interacting at the Sun are accompanied by particularly energetic solar energetic particle (SEP) events. This chapter is based on 25 events. The Fermi/LAT temporal data were made available to the HESPERIA project by G. Share prior to their publication in a comprehensive paper (Share et al. 2017). The present chapter introduces the relevant process of emission and pre-Fermi observations of piondecay $\gamma$-rays (Sect. 8.2), and gives an overview of the Fermi/LAT observations (Sect. 8.3). Section 8.3 was prepared by Gerald Share and Ron Murphy. Related X-ray and radio observations and associated SEP events are presented in Sects. 8.4 and 8.5 , respectively. Preliminary conclusions on the interpretation of the $\gamma$-ray events are in Sect. 8.6.

\subsection{Theory and Early Observations of Gamma-Ray Emission at Photon Energies $>60 \mathrm{MeV}$}

On 1982 Jun 3 the gamma-ray spectrometer on the Solar Maximum Mission satellite observed emission from 0.3 to $100 \mathrm{MeV}$ from a X8.0 GOES-class flare (Forrest et al. 1986). The impulsive flare lasted about $1 \mathrm{~min}$ and was followed by a distinct harder emission phase that peaked in about $1 \mathrm{~min}$ and lasted for over $15 \mathrm{~min}$. The energy spectrum of this sustained emission displayed a characteristic hump at photon energies above $60 \mathrm{MeV}$ (Fig. 8.1a), which appeared to be consistent with that from pion-decays produced by the interaction of $>300 \mathrm{MeV}$ protons in the solar atmosphere (Forrest et al. 1985, see below); the authors speculated whether the emission might be associated with the acceleration of solar energetic particles. There were several more of these events in the ensuing years, all associated with intense $\mathrm{X}$-class flares, that were summarized in a paper entitled "Long-Duration Solar Gamma-Ray Flares" (LDGRFs) (Ryan 2000). Various origins were suggested, and a 



Fig. 8.1 (a) The $\gamma$-ray spectrum showing the pion-decay bump of the 1982 Jun 3 event, observed by SMM (Forrest et al. 1985). Credit: Forrest et al., Internat. Cosmic Ray Conf. 4, 146, 1985, courtesy W.T. Vestrand. (b) Time profile of the first sustained $\gamma$-ray event detected by the Compton Gamma-Ray Telescope (adapted from Kanbach et al. (1993); credit: Kanbach et al., A\&A Suppl. 97, 349, 1993, reproduced with permission OESO)

key question was whether such emission would be observed when the accompanying flare was weaker and did not produce impulsive gamma radiation.

Detection of such events continued with observations from GAMMA-1 (Akimov et al. 1992), the Compton Gamma-Ray Observatory (CGRO) (Mandzhavidze and Ramaty 1992; Kanbach et al. 1993; Dunphy et al. 1999), GRANAT (Debrunner et al. 1997; Vilmer et al. 2003), CORONAS-F (Kuznetsov et al. 2011; Kuznetsov et al. 2014). Around 20 events had been observed then with significant emission above $60 \mathrm{MeV}$ from pion decay radiation (see Lockwood et al. 1997; Chupp and Ryan 2009; Vilmer et al. 2011 for reviews). For some of the events, pion decay radiation is observed during the impulsive phase of the event as defined by the production of hard X-rays above $100 \mathrm{keV}$. In some events, high energy emissions had also been observed for hours after the impulsive phase of the flare, revealing that high energy ions are present on time scales of several hours (e.g. Kanbach et al. 1993; Ryan et al. 1994; Ryan 2000; Rank et al. 2001).

\subsubsection{Pion-Decay $\gamma$-Ray Emission}

High-energy ( $>60 \mathrm{MeV}$ ) emission in solar flares results from nuclear interactions of mildly relativistic ions (above a few hundred $\mathrm{MeV} /$ nucleon) with the ambient solar atmosphere (most probably the dense chromosphere). These nuclear reactions result in the production of pions. Charged pions decay to electrons and positrons, which produce $\gamma$-ray emissions through bremsstrahlung radiation. Energetic positrons also 
contribute to the $\gamma$-ray continuum by annihilating in flight. The neutral pions decay in two photons, one being emitted at high energies. The result is a very flat spectrum with a broad-bump feature with a maximum at $67 \mathrm{MeV}$ (Murphy et al. 1987). In a magnetized region, synchrotron losses of electrons and positrons may be important and reduce their contributions with respect to the radiation from neutral-pion decay (Murphy et al. 1987; Vilmer et al. 2003). When energetic electrons are present at energies above $10 \mathrm{MeV}$, they produce bremsstrahlung emission above $10 \mathrm{MeV}$ (Chap. 2), which may mask pion-decay radiation.

Early work on the modelling of pion decay radiation and $\gamma$-ray emission (produced by $>10 \mathrm{MeV}$ ions) (Murphy et al. 1997) gave a first determination of the high energy spectrum of ions in flares. Assuming a spectral shape for the energetic ions (either a Bessel function or a power law), number and spectra of energetic protons were estimated for both the impulsive and the extended phase of the first detected event (1980 Jun 21) and showed that the proton spectrum was steeper in the impulsive phase than in the later, so-called 'extended' phase. This evolution of the ion spectrum in the extended phase was confirmed by further observations such as the ones of the 1991 Jun 11 event by CGRO/EGRET (Dunphy et al. 1999).

Quantitative analysis of several events with significant pion production has been performed providing information on the ion energy spectrum above $300 \mathrm{MeV} /$ nucleon and allowing a comparison of this spectrum with the one deduced at lower energies from $\gamma$-ray line spectroscopy (see, e.g., Alexander et al. 1994; Dunphy et al. 1999; Kocharov et al. 1994, 1998; Vilmer et al. 2003). These comparisons have shown that the ion energy distribution does not have a simple power-law form from the $\gamma$-ray line producing energy domain (1-10 MeV) to the pion-producing energy domain (>300 MeV/nucleon).

\subsubsection{Long-Duration $\gamma$-Ray Events}

Even before the Fermi era, a few events had been observed where enhanced piondecay radiation lasted several hours, and the question of the origin of these long duration events had been examined. On 1991 Jun 11 (Fig. 8.1b) emission above $50 \mathrm{MeV}$ was detected for almost $8 \mathrm{~h}$ after the flare by COMPTON/EGRET. Several interpretations had been proposed to explain these long duration emissions, either the continuous acceleration of protons above $300 \mathrm{MeV}$ (e.g., Ryan and Lee 1991) or the trapping of protons on very long time-scales. In particular, Mandzhavidze and Ramaty (1992) showed that the long duration phase could be explained by the injection of energetic protons in the impulsive phase and subsequent trapping. An efficient trapping on such long timescales required a strong mirror ratio in the trapping region $(>10)$ as well as a coronal density less than $5 \cdot 10^{11} \mathrm{~cm}^{-3}$. The question of how trapped particle populations could remain stable over hours was, however, not explained satisfactorily. An observational justification of continuous time-extended acceleration came from the discovery of sustained $\gamma$-ray events of moderate duration (1-2h), which were accompanied by non-thermal microwave emission (Kocharov et al. 1994; Trottet et al. 1994; Akimov et al. 1996). 


\subsection{New Insights of Sustained Emission Events from Fermi Observations}

With the launch of the sensitive Fermi Large Area Telescope (LAT) (Atwood et al. 2009), it became possible to observe weak $\gamma$-ray emission from the Sun due to its large effective area and aperture, and excellent background rejection. This permitted detection of quiescent $\gamma$-ray emission from cosmic-ray protons interacting in the solar atmosphere and cosmic-ray electrons interacting with sunlight (Abdo et al. 2011) with fluxes of 5 and $7 \times 10^{-7} \gamma \mathrm{cm}^{-2} \mathrm{~s}^{-1}$, respectively. LAT first detected transient $>100 \mathrm{MeV}$ solar $\gamma$-rays on 2010 Jun 12 from the impulsive M2 class flare (Ackermann et al. 2012a,b) during an $\sim 50$ s period. The emission was delayed about $10 \mathrm{~s}$ from the associated hard X-ray and nuclear-line emission and there was no evidence for any $>100 \mathrm{MeV} \gamma$-ray emission in the hours after the flare. The Fermi/LAT team reported the detection of $18>100 \mathrm{MeV}$ events associated with solar flares covering the time period from 2008 August to 2012 August (Ackermann et al. 2014) which they classified as being impulsive, sustained, or delayed. In some cases they categorized the events as having both impulsive and sustained characteristics. Only three of the events were classified as only having an impulsive component. We prefer to use the name "sustained" to categorize all emission that is distinct from the impulsive flare independent of its duration.

Details of the 2011 Mar 7 and 2011 Jun $7 \gamma$-ray observations and related solar measurements were also presented in Ackermann et al. (2014). The March 7 event was reported as having both impulsive and sustained emission components, while the June 7 event was classified as only having sustained emission because $\gamma$-rays were detected in only one LAT exposure about $1 \mathrm{~h}$ after the flare and there was no exposure to the flare. The $\gamma$-ray spectra were fit by two empirical models, a power law and a power law with exponential cutoff; and a physical pion-decay spectrum based on Murphy et al. (1987). Only heuristic arguments were presented to justify that the observed spectrum was from pion decay and not from electron bremsstrahlung. There was clear evidence for spectral softening over the $13 \mathrm{~h}$ period that the March 7 event was observed. As LAT's instrumental point spread function is about $1^{\circ}$, even above $1 \mathrm{GeV}$, only the location of the centroid of the $\gamma$-ray source could be deduced. The centroids of both sources were consistent with the active regions with uncertainties on the scale of a solar quadrant.

Ajello et al. (2014) reported detection on 2012 Mar 7 by LAT of what appeared to be distinct impulsive and sustained-emission phases associated with X5.4 and X1.3 (actually about M7 when the tail of the X5 flare is subtracted) flares, CMEs with speeds of about 2700 and $1800 \mathrm{~km} \mathrm{~s}^{-1}$, and a strong solar energetic particle event. The $\gamma$-ray flux was one of the brightest observed by LAT; it lasted close to $20 \mathrm{~h}$ and the fitted spectrum again softened with time. The time integrated centroid of the emission was consistent with the location of the flares with a $1 \sigma$ uncertainty of $\sim 10^{\circ}$. There is evidence that the source of the emission moved from the eastern to the western hemisphere about $7 \mathrm{~h}$ following the flares. Once again there was no information on the spatial extent of the emission. 
With detection of $>100 \mathrm{MeV} \gamma$-ray emission associated with two behind-thelimb solar flares, it has become clear that emission can extend as much as $40^{\circ}$ from the flare site (Pesce-Rollins et al. 2015; Ackermann et al. 2017). The events occurred on 2013 Oct 11 and 2014 Sep 1 and were associated with fast CMEs and strong solar energetic particle events. The centroids of the $\gamma$-ray emission for both events were close to the solar limb. The centroid for the October 11 event was consistent with the N21 latitude of the active region, located about $13^{\circ}$ behind the East limb, and its 1 $\sigma$ uncertainty extended to about $50^{\circ}$ East. In contrast, the centroid of the September 1 event was significantly north $\left(\sim 15^{\circ}\right)$ of the flare site that was located about $35^{\circ}$ behind the East limb.

More information on the characteristics and origin of these solar $\gamma$-ray events became available with the completion of a comprehensive study of 29 sustained emission events observed by LAT between 2008 and 2016 (Share et al. 2017). This study indicates that the emission is not spatially distributed globally on the Sun but is probably distributed over a few tens of degrees around the centroid location. From this study it is also clear that the sustained $>100 \mathrm{MeV}$ emission is temporally distinct from the impulsive flare phase, which often emits hard X-rays extending only to hundreds of $\mathrm{keV}$. The time profile of the first event observed by LAT on 2011 Mar 7, discussed above and plotted in Fig. 8.2, provides an example of such a distinct sustained-emission phase. While the analysis done by Share et al. (2017) is different from that of Ackermann et al. (2014), it uses the same source-class data and yields comparable fluxes and spectral results. It is simply a 'light-bucket' in which photons arriving with directions $<10^{\circ}$ of the Sun are accumulated, as long as the Sun is far from the Earth's horizon. The main plot in Fig. 8.2 shows the hours-long time profile of this $13 \mathrm{~h}$ event with LAT solar exposures every $3 \mathrm{~h}$. The background level contains roughly equal contributions from the quiescent Sun, and Galactic and extra-galactic $\gamma$ rays. The emission began during the time of the GOES flare and rose to peak about $6 \mathrm{~h}$ later.

The inset in Fig. 8.2 shows $>100 \mathrm{MeV}$ fluxes plotted at 4 min resolution after the hard X-ray peak of the March 7 flare. For comparison, the dotted line follows the high-energy time profile observed in the 1991 June 11 event which exhibited both impulsive emission and sustained emission lasting $8 \mathrm{~h}$. The $>100 \mathrm{MeV}$ emission on March 7 appears to increase during the observations after the impulsive phase. There is also no evidence for nuclear line emission during the impulsive flare, suggesting that $>1 \mathrm{MeV}$ protons were not present in significant numbers. A limit on the 2.223 MeV neutron capture line during the flare suggests that the flare had at most only $20 \%$ of the number of $>500 \mathrm{MeV}$ protons observed in the sustained emission phase (Share et al. 2017). This indicates that only sustained $>100 \mathrm{MeV}$ emission was observed by LAT on March 7, in contrast to what was reported by Ackermann et al. (2014). The same conclusion can be reached about other events reported by Ackermann et al. (2012b) to have impulsive $>100 \mathrm{MeV}$ phases. Specifically, Share et al. (2017) find that the first $>100 \mathrm{MeV}$ outburst on 2012 Mar 7 described in detail by Ackermann et al. (2014) was distinct sustained emission and not directly associated with the X5.4 flare. 


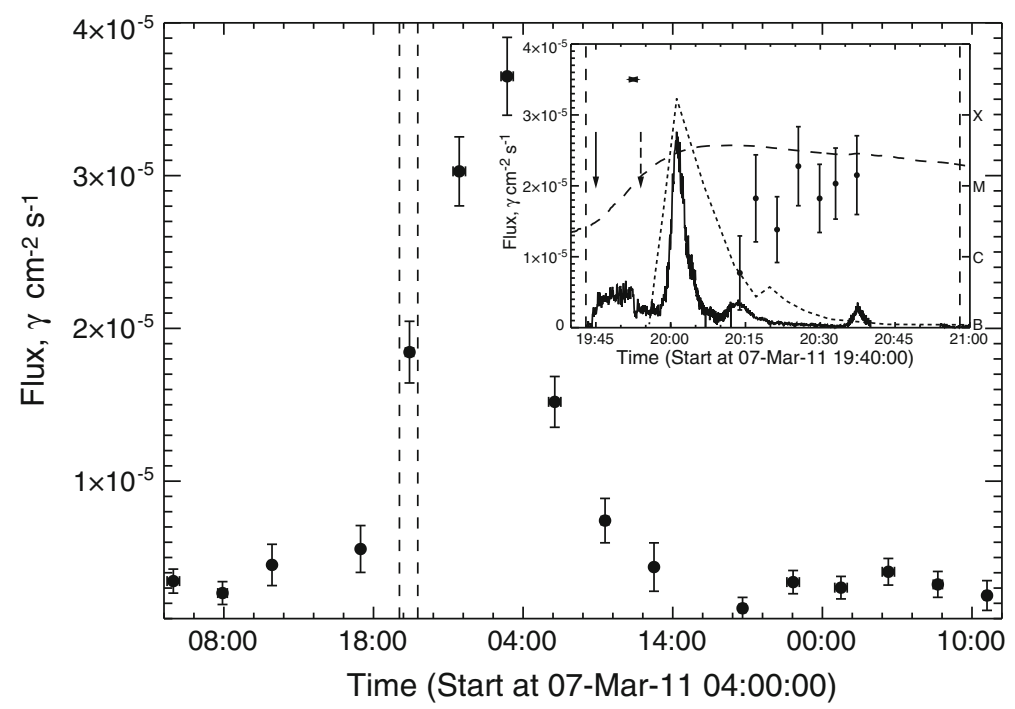

Fig. 8.2 Time history of the $>100 \mathrm{MeV}$ flux from $\leq 10^{\circ}$ of the Sun revealing the $2011 \mathrm{Mar} 7$ LAT event (data points and $\pm 1 \sigma$ statistical uncertainties). Vertical dashed lines show the GOES start and end times. The inset shows 4-min accumulation LAT data points and the merged and arbitrarily scaled $>100 \mathrm{keV}$ count rates observed by RHESSI and Fermi/GBM during the impulsive

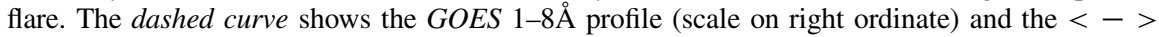
symbol shows the range in CME onset times in the CDAW catalog derived for linear and quadratic extrapolations. The vertical solid arrow depicts our estimate of the CME onset from inspection of SDO/AIA images and the vertical dashed arrow shows the estimated onset of Type II radio emission

Share et al. (2017) also find that 19 of the 29 sustained-emission events had time profiles distinct from the impulsive phase and most of these had onset times after the hard X-ray peaks. For the remaining events, there were not enough data to determine whether the sustained emission was distinct from or primarily associated with the impulsive phase, but in no case is there clear evidence that the sustained emission is the tail of the impulsive flare.

Pion-decay spectra from protons having different power-law spectral indices fit the spectra from the 2011 Mar 7 event and the other 28 events (Share et al. 2017). In general the results are consistent with those obtained by Ackermann et al. (2014, 2017) and Ajello et al. (2014). In addition, Share et al. (2017) demonstrated that only pion-decay spectra are consistent with the brightest sustained emission events and that any plausible electron bremsstrahlung spectra are not. Using nuclear line observations during these same bright sustained emission events, it was also possible to demonstrate that the proton spectra from 10 to $300 \mathrm{MeV}$ were flatter than the spectra at higher energies. In addition to the two events found to have spectra that softened with time, Share et al. (2017) found two more that softened in time and two that hardened in time. 
Detailed spectroscopic studies of many of the events observed by LAT revealed that the number of $>500 \mathrm{MeV}$ protons producing the sustained emission was typically at least a factor ten more than found in the accompanying impulsive flare. This is consistent with the distinctly different nature of the time profiles of the impulsive flares and the sustained emission discussed above. As Share et al. (2017) discuss, it is now clear that another energy source is necessary to accelerate protons to energies $>300 \mathrm{MeV}$ in order to produce the pion-decay emission observed in the sustained events. These energetic considerations and the rise in sustained $\gamma$ ray emission following the impulsive phases in many events suggests the likely source of the energy is the accompanying fast CME, possibly through its shock that is thought to produce gradual SEPs. The authors (Share et al. 2017) find that the number of $>500 \mathrm{MeV}$ SEP protons is on average about 100 times the number returning to the Sun to produce the sustained $\gamma$-ray emission. This is consistent with what shock wave models estimate (Kocharov et al. 2015).

The 2013 Oct 13 and 2014 Sep 1 behind-the-limb events (Ackermann et al. 2017) were also studied by Share et al. (2017). The time profile of the latter event, which lasted at least $2 \mathrm{~h}$, is plotted in Fig. 8.3. Both $>100 \mathrm{MeV} \gamma$-ray and $>100 \mathrm{keV}$ electron bremsstrahlung rose about $7 \mathrm{~min}$ following the impulsive hard X-ray flare, as inferred from SAX observations on MESSENGER (Schlemm et al. 2007). The bremsstrahlung extended up to at least $10 \mathrm{MeV}$ and is consistent with production by

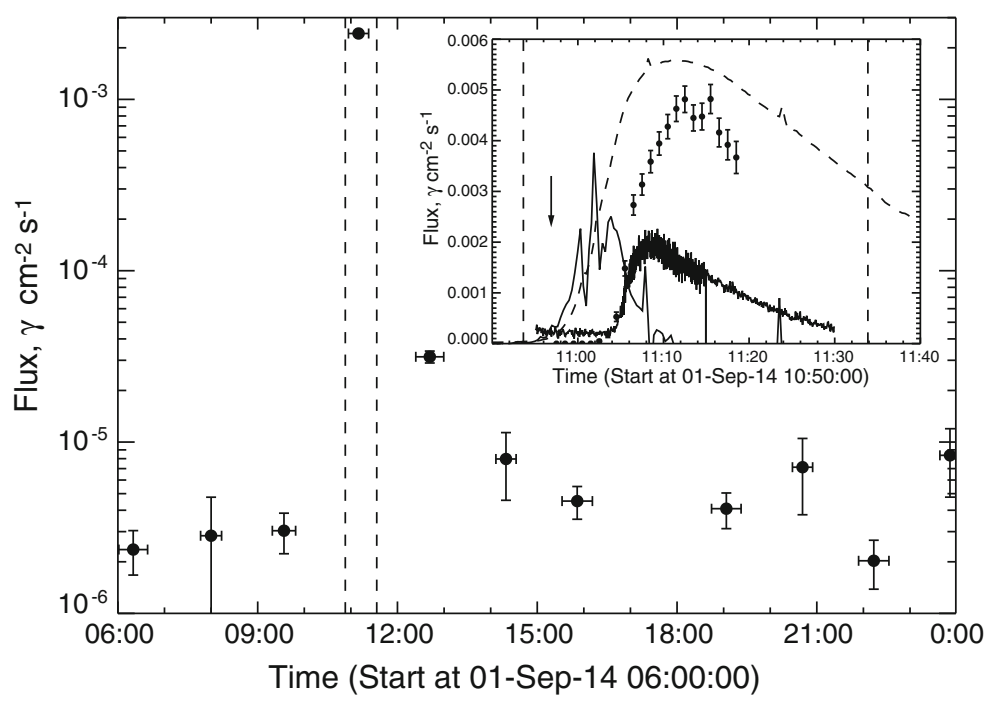

Fig. 8.3 Time history of the $>100 \mathrm{MeV}$ flux of the Sun revealing the 2014 Sep 1 LAT event (data points and $\pm 1 \sigma$ statistical uncertainties). Vertical dashed lines show the soft X-ray start and end times from MESSENGER/SAX. The inset shows 1-min accumulation $>100 \mathrm{MeV}$ data points $( \pm 1 \sigma$ statistical uncertainties) and the arbitrarily scaled $100-300 \mathrm{keV}$ count rates observed by GBM. The dashed curve shows the soft X-ray profile from SAX and the thin solid curve its derivative. The vertical solid arrow depicts our estimate of the CME onset from inspection of SDO/AIA images 
electrons interacting in a thick target, indicating the electrons were produced by the same CME shock that accelerated the protons producing the sustained $\gamma$ radiation (Share et al. 2017). This conclusion is consistent with the finding that a direct magnetic connection exists between the shock wave and the low solar atmosphere at the onset of the hard X-rays and $\gamma$-rays in both behind the limb events (Plotnikov et al. 2017).

The conclusion reached by Share et al. (2017) is that the sustained emission events are likely due to shock-accelerated particles, associated with those in SEPs, that are imparted to field lines that return to the Sun and those that return to the Sun on open field lines from an SEP reservoir. This interpretation will be discussed in Sect. 8.4.

\subsection{Multiwavelength Observations of Fermi/LAT $\gamma$-Ray Events}

In this section we examine the relationship between the $\gamma$-ray events and other manifestations of energy release in the corona, namely heating as revealed by soft X-ray bursts, and electron acceleration traced by hard X-ray and radio emission. We use especially hard X-ray observations from RHESSI (Lin et al. 2002) and INTEGRAL/ACS (Rodríguez-Gasén et al. 2014), whole-Sun radio fluxes from the RSTN and Nobeyama Observatories (Torii et al. 1979; Nakajima et al. 1985) on ground, and from the radio spectrographs aboard Wind (Bougeret et al. 1995) and STEREO (Bougeret et al. 2008).

At decametric and longer waves (referred to as DH in the following) all $25 \gamma$-ray events were accompanied by type III bursts, and $23 / 25$ by type II bursts. The type III bursts show that electrons (particles) had access to open field lines during all $\gamma$-ray events. The bursts occurred in general during the impulsive and early post-impulsive phase of the parent flare, as commonly observed in SEP events. They were not found later on during long-duration $\gamma$-ray events. The presence of type II bursts shows that shock waves in the high corona are a common counterpart of $\gamma$-ray events. Metric continuum emission (type IV bursts) is observed in some events.

\subsubsection{Impulsive and Early Post-impulsive $\gamma$-Ray Emission}

A rare $\mathrm{Fermi} / \mathrm{LAT}$ observation showing a $\gamma$-ray event during and after the impulsive flare phase is illustrated in Fig. 8.4. Hard X-rays (INTEGRAL/ACS, photon energies $>80 \mathrm{keV}$; Rodríguez-Gasén et al. 2014) rise and decline during the rise of the soft X-ray flux, as do the radio flux densities at 15,400 and $8800 \mathrm{MHz}$. The $\gamma$-ray emission, which is observed with much poorer time coverage, shows a similar rise and initial decay, but then stays on an enhanced level until the end of 


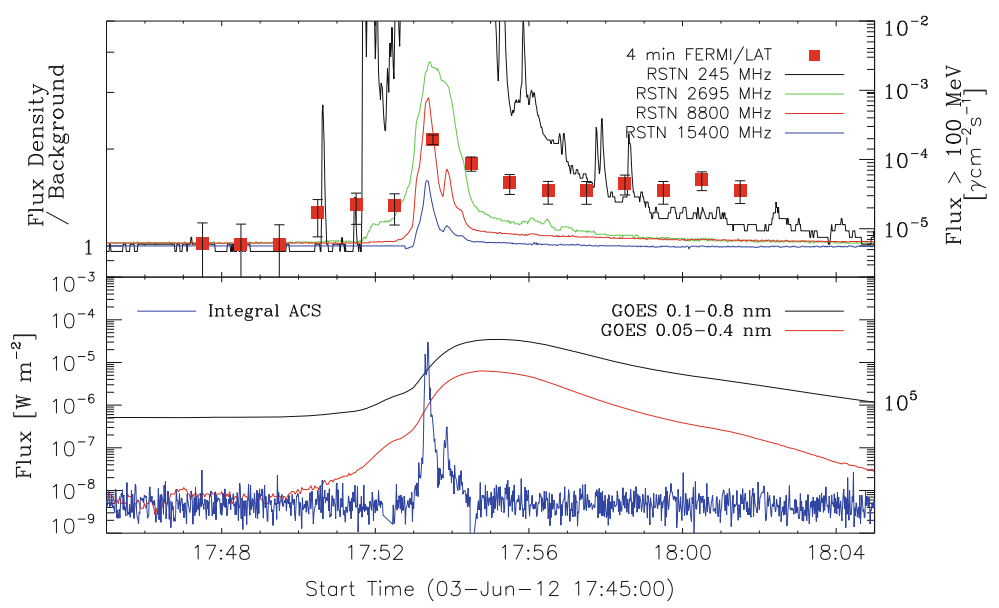

Fig. 8.4 Time evolution of the high-energy $\gamma$-ray, X-ray and radio emission in the impulsive and early post-impulsive phase of a flare

the observations. This long persistence is the key feature of the sustained events introduced in Sect. 8.3. While the microwaves $\geq 8800 \mathrm{MHz}$ show no counterpart, emissions at 2695 and $245 \mathrm{MHz}$ have a prolonged tail that accompanies the observed part of the post-impulsive $\gamma$-ray emission. Renewed energy release after the impulsive phase is also suggested by the bump in the decaying soft $\mathrm{X}$ ray profile. The continued emission of both pion-decay $\gamma$-rays and microwaves in the early post-impulsive phase is consistent with the earlier observations by GAMMA1 (Kocharov et al. 1994; Akimov et al. 1996) and SMM (Trottet et al. 1994).

\subsubsection{Long-Duration $\gamma$-Ray Events}

As shown in Fig. 8.2, $\gamma$-ray emission may rise again well after the impulsive phase. Among the $25 \gamma$-ray events studied, a total of twelve had duration longer than $2 \mathrm{~h}$. Long-duration $\gamma$-ray emission occurs most often during the decay of the associated soft X-ray burst, as illustrated in Fig. 8.5. In EUV images taken by AIA/SDO or SWAP/Proba2 the decay of the soft X-ray bursts is accompanied by the formation of post-flare loop arcades.

Although there is still some microwave burst activity at low frequencies (2.695 MHz) in Fig. 8.5, the long-duration $\gamma$-rays are in general not accompanied by time-extended or recurrent hard X-ray or microwave burst activity. On $2011 \mathrm{Mar}$ 7 the long-duration $\gamma$-rays are accompanied by several flares, rather than the decay of a single soft X-ray burst. But the microwave or hard X-ray emissions are weak, and in any case are only observed during a short fraction of the $\gamma$-ray event. In 


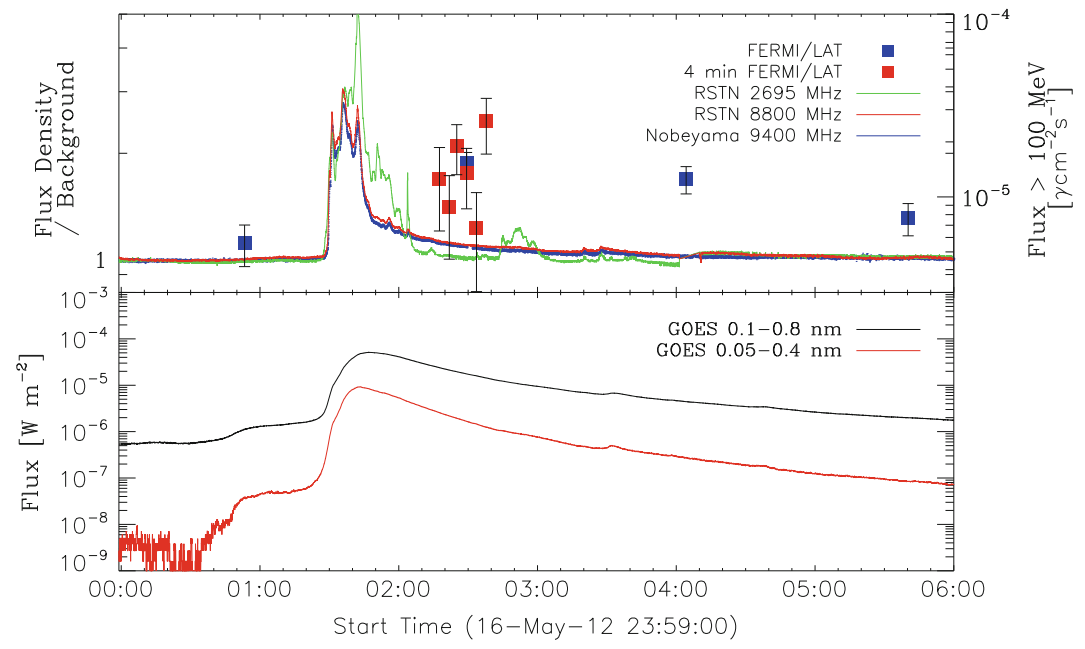

Fig. 8.5 Time evolution of the high-energy $\gamma$-ray, soft and hard X-ray and radio emission from the impulsive phase throughout the rise and decay of sustained $\gamma$-ray emission

summary, we find no evidence of repeated efficient electron acceleration in the low corona during most parts of this or any other long-duration $\gamma$-ray event.

\subsubsection{Soft X-Ray Bursts and $\gamma$-Ray Events}

The close relationship between the durations of $\gamma$-ray and soft X-ray emissions is confirmed by the quantitative analysis of the decay profiles of soft X-ray bursts associated with $\gamma$-ray events: short duration $\gamma$-ray bursts $(<2 \mathrm{~h}$; the exact duration is difficult to determine because of gaps in the solar observations of Fermi/LAT) are found to have relatively short soft X-ray decay phases, while the long $\gamma$-ray events accompany comparatively long soft X-ray decay phases. There are some intermediate events, showing the distribution of burst durations is not bimodal. But it is clear from this observational analysis that long-duration $\gamma$-ray events tend to be associated with long-duration energy release in the solar corona.

Although the long-duration $\gamma$-ray emission may start more than an hour after the peak of the associated soft X-ray burst, the logarithms of the peak fluxes of the two emissions correlate, with a linear correlation coefficient 0.72 , and a probability that the same or a higher value is obtained from two unrelated samples of $0.8 \%$. The corresponding values for the CME speed and the logarithm of the $\gamma$-ray peak flux are 0.61 and $3.7 \%$, respectively. These are rough evaluations, because the $\gamma$-ray time profile is not densely covered by the Fermi/LAT observations, and the peak fluxes are only lower limits. 




Fig. 8.6 Time history of high-energy $\gamma$-rays and the decametric-hectometric radio emission, showing a type II burst accompanies the $\gamma$-rays. The grey vertical stripes in the bottom panel show time intervals of unfavourable solar observing conditions for Fermi/LAT

\subsubsection{Coronal Shock Waves and $\gamma$-Ray Events}

The only signature of electron acceleration that we found to accompany long-lasting $\gamma$-ray emission are DH type II bursts, which are ascribed to CME-driven shocks (Reiner et al. 2007). The type II burst in Fig. 8.6 mostly shows up as a sequence of patches after $\sim 7$ UT that gradually drift from higher to lower frequencies. The noticeable fact is that this emission persists throughout the several hours duration of the $\gamma$-ray emission.

It is tempting to interpret the association between sustained $\gamma$-ray events and $\mathrm{DH}$ type II bursts as evidence that the CME shock accelerates protons to relativistic energies, which then stream back from the downstream region of the shock to the low solar atmosphere, where they create pions (see Sect. 8.3 and Chap. 9). We consider in the following the implication of this interpretation on the number of particles that must be present in the downstream region of the shock in order to account for the observed $\gamma$-ray emission. To this end we analyze the events with a long duration sustained emission that occurred on 2011 Mar 7, 2012 Jan 23, Mar 5 and May 17.

All four events were accompanied by CMEs well-observed by the LASCO coronagraphs. The quasi-parallel shock often invoked as the site of SEP acceleration is expected to be located around the summit of the CME, following the trajectory 
shown by the height-time curves given in the LASCO CME catalogue. ${ }^{1}$ The type II source is usually believed to be located in the quasi-perpendicular region of the shock (Pulupa and Bale 2008), hence on the flank of the CME rather than near its summit. We therefore considered separately the heights of the CME estimated by the LASCO catalogue and of the type II source, inferred from the electron density model of Leblanc et al. (1999), both at the peak of the long-duration $\gamma$-ray emission. The heliocentric distances of the CME apices range from $11 \mathrm{R}_{\odot}$ to $26 \mathrm{R}_{\odot}$, those of type II sources from $7 \mathrm{R}_{\odot}$ to $18 \mathrm{R}_{\odot}$.

Particles accelerated at the shock must hence stream to the solar chromosphere over a distance of many solar radii without being mirrored by the magnetic field before. This implies that they are injected into coronal magnetic field lines near the shock with a pitch angle smaller than some limiting value $\alpha_{0}$ such that $\sin ^{2} \alpha_{0}=$ $B(r) / B\left(R_{\odot}\right)$. The magnetic field decreases outward from the low corona with some power of the heliocentric distance $r$. If we just extrapolate the radial component of the solar wind magnetic field back to the photosphere, hence assuming that $B(r) \sim r^{-2}$, the maximum pitch angles allowed for protons that reach the low solar atmosphere are $2^{\circ}-5^{\circ}$ when the particles start from the CME apex, and $3^{\circ}-8^{\circ}$ when they start from the type II source. Hence only protons injected nearly parallel to the coronal magnetic field lines near the shock can reach a sufficiently dense part of the solar atmosphere to undergo nuclear reactions. If we suppose that the protons are isotropically distributed at the shock, only a fraction $1-\cos \alpha_{0}$ is expected to achieve this. This fraction is $1 \%$ of the initial population for $\alpha_{0}=8^{\circ}\left(6 \cdot 10^{-4}\right.$ for $\left.\alpha_{0}=2^{\circ}\right)$. It will be further reduced by the expected stronger decrease of the magnetic field within the solar wind source surface.

\subsection{Solar Energetic Particle Events Associated with Fermi/LAT Gamma-Ray Events}

All Fermi/LAT $\gamma$-ray events discussed in Sect. 8.3 are associated with SEP events observed at $1 \mathrm{AU}$ from the Sun, by instruments at L1 or by the twin STEREO spacecraft, or both. Proton and/or electron enhancements are observed with SoHO/ERNE (Torsti et al. 1995), STEREO/LET (Mewaldt et al. 2008), STEREO/HET (von Rosenvinge et al. 2008), STEREO/SEPT (Müller-Mellin et al. 2008), and ACE/EPAM (Gold et al. 1998) over a wide range of energy (55 keV to $4 \mathrm{MeV}$ for electrons and 1.6 to $130 \mathrm{MeV}$ for protons). Higher energy protons are available from SoHO/EPHIN (penetrating protons at $100-1000 \mathrm{MeV}$ energies) as well as from GOES/HEPAD (only for 9 of the 25 investigated Fermi/LAT events, see Fig. 8.7 for an example) in three differential energy channels ranging from 330 to $700 \mathrm{MeV}$ and an integral channel $>700 \mathrm{MeV}$.

\footnotetext{
${ }^{1}$ https://cdaw.gsfc.nasa.gov/CME_list/.
} 


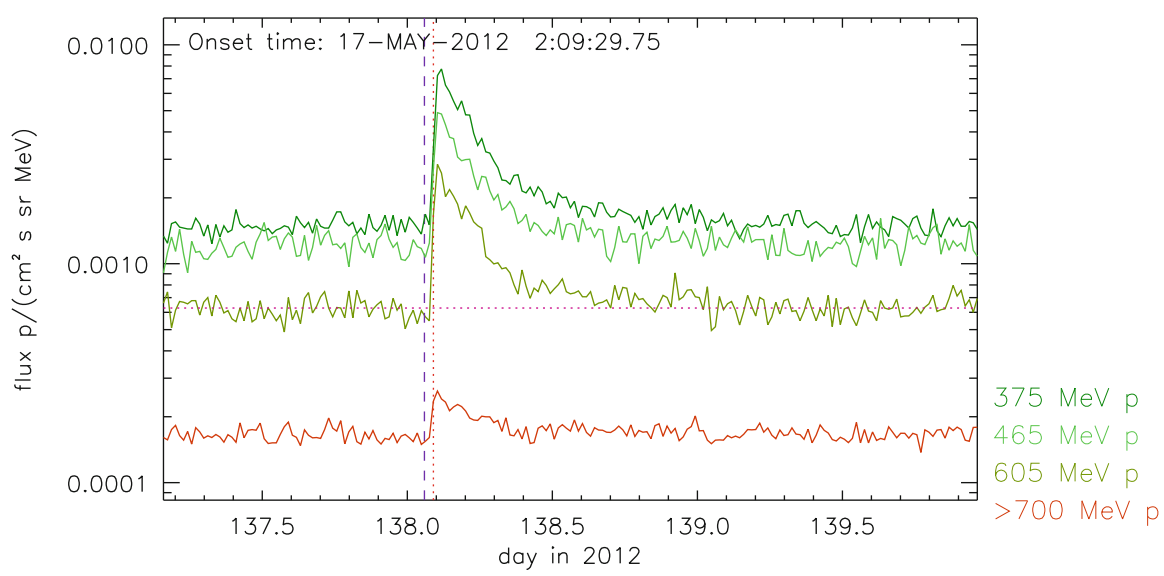

Fig. 8.7 GOES 15/HEPAD proton fluxes on 2012 May 17. The vertical purple dashed and the red dotted lines indicate respectively the flare onset and the derived SEP onset with the sigma-method (see text)

\subsubsection{SEP Characteristics and Association with Fermi/LAT}

Most of the SEP events show a slow rise phase, but some show a fast rise phase in all observed energy ranges (see Reames 1999 for a review on general SEP characteristics). This distinction is somewhat subjective, but we mean by "fast" a rise in 2-3 h, and by "slow" a rise within several hours, sometimes one day. Onset times for all observed SEP events can be derived either by implementing a threshold to be exceeded by the flux as described in Malandraki et al. (2012) or a PoissonCUSUM analysis (Huttunen-Heikinmaa et al. 2005). For SEP events with a fast rise a velocity dispersion analysis (VDA) can be used (Krucker et al. 1999; Malandraki et al. 2012) to estimate both the release time at the Sun and the apparent path length of the particles: the solar release time of the first particles seen at the spacecraft is obtained from a linear fit of the derived onset times of electrons and protons at different energies as a function of their respective inverse velocity. Such an analysis could only be performed for 11 of the $25 \mathrm{Fermi} / \mathrm{LAT}$ events. For a comparison of derived release times with photon arrival times measured at the observer's distance (1 AU) we add $8 \mathrm{~min}$.

Hereafter, we will discuss the events of 2011 Mar 7, 2012 Mar 7, 2013 Apr 11 and 2014 Feb 25, for which a VDA analysis could be performed at L1 and/or STEREO, as well as the events of 2012 Jan 23 and May 17 that are of particular interest for numerical transport simulations discussed in Chap.9. Table 8.1 gives the date and location, soft X-ray class, start and peak time, start and end of the decametricto-hectometric type III bursts and CME speed from the LASCO/CME catalogue. SEP parameters are given for STEREO A and B (STA, STB), for SoHO/ERNE or ACE/EPAM at L1, and for GOES/HEPAD (HEP) such as the characterization of the 
Table 8.1 Characteristics of the 6 SEP event cases discussed in this section (see text for further information)

\begin{tabular}{|c|c|c|c|c|c|c|}
\hline \multirow[b]{2}{*}{ Date } & \multicolumn{2}{|c|}{ Soft X-rays } & \multirow[b]{2}{*}{ DH III } & \multirow[b]{2}{*}{$\begin{array}{l}\text { CME } \\
{\left[\mathrm{km} \mathrm{s}^{-1}\right]}\end{array}$} & \multirow[b]{2}{*}{$\begin{array}{l}\text { SEP } \\
\text { (s/c, rise, energy) }\end{array}$} & \multirow[b]{2}{*}{$\begin{array}{l}\mathrm{SRT}+8 \mathrm{~min} \\
{[\mathrm{~min}]}\end{array}$} \\
\hline & $\begin{array}{l}\text { Class } \\
\text { Location }\end{array}$ & $\begin{array}{l}\text { Start } \\
\text { Peak }\end{array}$ & & & & \\
\hline \multirow[t]{4}{*}{2011 Mar 7} & M3.7 & $19: 43$ & \multirow[t]{4}{*}{$19: 50-20: 10$} & \multirow[t]{4}{*}{2125} & $\mathrm{STA}(\mathrm{s}) \sim 60 \mathrm{MeV}$ & - \\
\hline & \multirow[t]{3}{*}{ N30W47 } & \multirow[t]{3}{*}{$20: 58$} & & & $\mathrm{~L} 1(\mathrm{f}) \sim 80 \mathrm{MeV}$ & $20: 26 \pm 00: 04$ \\
\hline & & & & & HEP no detection & - \\
\hline & & & & & STB (f) $\sim 60 \mathrm{MeV}$ & - \\
\hline \multirow[t]{4}{*}{23 Jan 2012} & M8.7 & 03:38 & \multirow[t]{4}{*}{$03: 40-04: 20$} & \multirow[t]{4}{*}{2175} & STA $(s) \sim 100 \mathrm{MeV}$ & - \\
\hline & \multirow[t]{3}{*}{ N33W21 } & \multirow[t]{3}{*}{$04: 34$} & & & $\mathrm{~L} 1$ (f) $\sim 100 \mathrm{MeV}$ & - \\
\hline & & & & & $\begin{array}{l}\mathrm{HEP}(\mathrm{s}) \sim 600 \mathrm{MeV} \\
\text { weak }\end{array}$ & - \\
\hline & & & & & STB $(s) \sim 100 \mathrm{MeV}$ & - \\
\hline \multirow[t]{4}{*}{2012 Mar 7} & X5.4 & 00:02 & \multirow[t]{2}{*}{$00: 15-01: 00$} & \multirow[t]{2}{*}{2864} & $\mathrm{STA}(\mathrm{s})>130 \mathrm{MeV}$ & - \\
\hline & N17E27 & 00:40 & & & $\mathrm{L} 1(\mathrm{~s})>100 \mathrm{MeV}$ & $23: 21 \pm 00: 51$ \\
\hline & $\mathrm{X} 1.2$ & 01:05 & \multirow[t]{2}{*}{$01: 15-01: 30$} & \multirow[t]{2}{*}{1825} & $\mathrm{HEP}(\mathrm{s})>700 \mathrm{MeV}$ & - \\
\hline & N17E27 & $01: 23$ & & & $\mathrm{STB}(\mathrm{f})>100 \mathrm{MeV}$ & 00:37士00:01 \\
\hline \multirow[t]{4}{*}{2012 May 17} & M5.1 & $01: 25$ & \multirow[t]{4}{*}{$01: 30-01: 40$} & \multirow[t]{4}{*}{1582} & STA $(\mathrm{s}) \sim 100 \mathrm{MeV}$ & - \\
\hline & \multirow[t]{3}{*}{ N07W88 } & \multirow[t]{3}{*}{ 02:14 } & & & $\mathrm{L} 1(\mathrm{f}) \sim 130 \mathrm{MeV}$ & - \\
\hline & & & & & $\operatorname{HEP}(\mathrm{f})>700 \mathrm{MeV}$ & - \\
\hline & & & & & $\mathrm{STB}(\mathrm{s}) \sim 100 \mathrm{MeV}$ & - \\
\hline \multirow[t]{4}{*}{2013 Apr 11} & M6.5 & $06: 55$ & \multirow[t]{4}{*}{ 07:00-07:30 } & \multirow[t]{4}{*}{861} & STA (s) weak & - \\
\hline & \multirow[t]{3}{*}{ N07E13 } & \multirow[t]{3}{*}{$07: 29$} & & & $\mathrm{~L} 1(\mathrm{f}) \sim 130 \mathrm{MeV}$ & 07:24士00:08 \\
\hline & & & & & $\begin{array}{l}\text { HEP very weak } \sim 600 \\
\mathrm{MeV}\end{array}$ & - \\
\hline & & & & & $\mathrm{STB}(\mathrm{f}) \sim 100 \mathrm{MeV}$ & 07:10土00:01 \\
\hline \multirow[t]{4}{*}{25 Feb 2014} & $\mathrm{X} 4.9$ & $00: 39$ & \multirow[t]{4}{*}{$00: 45-01: 15$} & 2147 & STA (f) $\sim 100 \mathrm{MeV}$ & 01:06土00:01 \\
\hline & N00E78 & 01:03 & & & $\mathrm{L} 1(\mathrm{~s}) \sim 130 \mathrm{MeV}$ & $01: 22 \pm 00: 20$ \\
\hline & & & & & HEP very weak & - \\
\hline & & & & & $\mathrm{STB}(\mathrm{f}) \sim 100 \mathrm{MeV}$ & 00:56士00:01 \\
\hline
\end{tabular}

SEP flux rise as "fast" (f) or "slow" (s), the approximate energy up to which the SEPs are observed and the derived solar release time (SRT) from VDA.

2011 Mar 7: The event had moderate SEP flux. The Fermi/LAT sustained emission (Fig. 8.2) was observed $\sim 15 \mathrm{~min}$ after the CME onset (defined from inspection of SDO/AIA images) and peaked several hours later $(\sim 7 \mathrm{~h})$. The derived SRT from the L1 observations is $\sim 20 \mathrm{~min}$ later than the Fermi/LAT emission onset and the particle path length of $\sim 1.9$ AU does not indicate substantial scattering.

2012 Jan 23: An intense SEP event was observed by ACE/EPAM at L1. VDA could not produce reliable SRT results, while GOES/HEPAD indicates a very weak event with a poorly determined onset $(\sim 7 \mathrm{~h}$ after the flare). The weak event was preceded by a slow rise right after the flare. The interaction between the parent CME and a preceding much slower one $\left(1400 \mathrm{~km} \mathrm{~s}^{-1}\right)$, associated with an earlier 
M1.1 class flare, may be responsible for both the observed high SEP flux and the significant changes in the SEP intensity profile (Joshi et al. 2013). Sustained Fermi/LAT emission was observed $\sim 15 \mathrm{~min}$ after the hard X-ray flare peak, lasting for many hours while the delayed observed GOES/HEPAD onset with respect to the Fermi/LAT onset could be related to this several-hours lasting sustained $\gamma$-ray emission.

2012 Mar 7: For the double solar event with considerable SEP flux, GOES/HEPAD saw a steep rise $\sim 6 \mathrm{~h}$ after the first flare. It was, however, preceded by a continuous slow rise starting immediately after the flare, as often observed in eastern SEP events. According to Kouloumvakos et al. (2016) (a) the first flare/CME was responsible for the SEP event observed at different spacecraft, (b) the proton SRT observed by STEREO B is consistent with the arrival of an observed EUV wave on the Sun at the STEREO-B footpoint, and (c) the considerably delayed SRT at L1 compared to STEREO B suggests a release of particles further away from the Sun consistent with the timing and location of the shock's western flank. No plausible explanations could be derived from the observed behaviour at STEREO A. Our L1 VDA analysis is not reliable as the derived apparent particle path length is $\sim 8$ AU. The observed large Fermi/LAT sustained fluxes, peaking after the first flare and lasting for several hours, have a derived first onset near the first flare peak time, while the second Fermi/LAT onset is estimated $\sim 45 \mathrm{~min}$ after the second flare onset. The derived STEREO B release time is co-temporal, within errors, with the first Fermi/LAT onset.

2012 May 17: The strong SEP event was associated with the first Ground Level Enhancement (GLE) of solar cycle 24. VDA could not produce reliable SRTs. GOES/HEPAD registered a fast rising intense event starting right after the flare (see Fig. 8.7). The expansion of the shock forming in the corona was studied using a new technique based on coronal magnetic field reconstructions, full magnetohydrodynamic simulations and multi-point imaging inversion techniques (Rouillard et al. 2016). This analysis concluded that $\mathrm{GeV}$ particles were released when this shock became super-critical (Mach numbers $>3$ ). The magnetic connectivity between the shock and L1 was established via a magnetic cloud that erupted from the same active region 5 days earlier. Fermi/LAT observations (Fig. 8.5) indicate significant sustained emission starting after the flare and lasting several hours, while the GOES/HEPAD flux onset coincides with the Fermi/LAT emission onset.

2013 Apr 11: A fast-rising strong SEP event was observed at L1 and STEREO B, but only a small slowly rising one at STEREO A. GOES/HEPAD recorded a very weak event with a poorly-determined onset $3 \mathrm{~h}$ after the flare. By determining the angular extent of the observed EUV wave and CME, Lario et al. (2014) concluded that while the particle SRT from STEREO B is within uncertainties consistent with the arrival of the EUV wave and CME-driven shock at the footpoint of the spacecraft, the EUV wave did not reach the footpoint of the field lines connecting to L1; the observed intense SEPs at L1 were most likely originating from the western flank of the CME-driven shock as it was propagating higher in the corona. Our analysis indicates (a) a particle release time to L1 that coincides within errors with the peak of the flare and is at least 15 min later than the SRT from STEREO B and 




Fig. 8.8 Fermi/LAT observations for the 2014 Feb 25 event. Vertical light and dark green lines indicate respectively the derived STEREO A and B onsets, while purple and orange vertical lines indicate the respective L1 and Fermi/LAT onsets. Horizontal lines indicate the derived error ranges

(b) an apparent path length of $\sim 2.1 \mathrm{AU}$, consistent with the picture emerging from Lario et al. (2014). The derived STEREO B SRT coincides precisely with the onset of the Fermi/LAT sustained emission that lasts for $\sim 20 \mathrm{~min}$ and peaks a few minutes after the soft X-ray flux.

25 Feb 2014 (Fig. 8.8): The SEPs rose fast to high fluxes at STEREO A and B, while a weak slowly-rising event was recorded at L1. GOES/HEPAD showed a very weak SEP event with a poorly-determined onset $>5 \mathrm{~h}$ after the flare. According to Lario et al. (2016), despite the considerable distance between the footpoints of the field lines connecting the Sun with STEREO A, B and L1 and the small extent of the observed EUV wave, the expansion of the extended shock, accompanying the $\mathrm{CME}$, to higher latitudes into the corona determined the release of particles and the observed intense SEP event. Our analysis indicates a SRT at both STEREO satellites and L1 well after the flare in accordance to these findings. However, the VDA at L1 and the respective SRT are not reliable as the particle path length is $~ 5.2 \mathrm{AU}$. Due to gaps in the observing time, sustained $\gamma$-ray emission is only observed for a short time, starting $\sim 15 \mathrm{~min}$ after the onset of the flare, then peaking and dropping quite rapidly within $\sim 20 \mathrm{~min}$. The STEREO B SRT coincides with the Fermi onset, while the STEREO A SRT is $10 \mathrm{~min}$ later.

\subsubsection{SEP Spectra}

Event-integrated energy spectra of oxygen, neon, and iron+nickel as a group, and the abundance ratios $\mathrm{Ne} / \mathrm{O},(\mathrm{Fe}+\mathrm{Ni}) / \mathrm{O}$, and $(\mathrm{Fe}+\mathrm{Ni}) / \mathrm{Ne}$ were investigated by using 
SOHO/ERNE measurements. Heavy ion signatures were searched for in all $25 \gamma$ ray events, and were observed above the quiet-time background in twelve of them. For events occurring in a close sequence of time it was not possible to distinguish possible multiple injections of heavy ions that could have been associated with each individual $\gamma$-ray event. This was the case, e.g., for the four events during the time period of 2012 Mar 7-10. The heavy ions observed were associated with the first $\gamma$-ray event of this period. Continuous data coverage over an entire event was also required, and this limited the investigation to eight of the $25 \gamma$-ray events.

The event-integrated intensities of $\mathrm{O}, \mathrm{Ne}$ and $\mathrm{Fe}+\mathrm{Ni}$ were calculated for 10 energy channels between $\sim 3.2-160 \mathrm{MeV} /$ nucleon of each ion species by integrating from the event onset in each energy channel till the time when the backgrounddetrended cumulative intensity at that energy reached $95 \%$ of the maximum. Thus, individual integration times were used for each ion at each energy channel. The energy spectra were fitted by using double power-law functions by Band et al. (1993). The fitting procedure gave the low and high-energy spectral indices $\left(\gamma_{\mathrm{a}}\right.$ and $\left.\gamma_{\mathrm{b}}\right)$, the break energy $\left(E_{\mathrm{B}}\right)$, and the normalization constant for the spectra. These quantities for oxygen and iron+nickel are given in Table 8.2 for the events of 2012 Mar 7 and 2013 Apr 11. The measured abundance ratios $\mathrm{Ne} / \mathrm{O}$ and $(\mathrm{Fe}+\mathrm{Ni}) / \mathrm{O}$ as function of energy are shown in Fig. 8.9. The $\gamma$-ray events were characterized by the peak fluxes of $\sim 94 \times 10^{-5} \mathrm{~cm}^{-2} \mathrm{~s}^{-1}\left(2012\right.$ Mar 7) and $\sim 3.9 \times 10^{-5} \mathrm{~cm}^{-2} \mathrm{~s}^{-1}$ (2013 Apr 11) with event durations of $9.9 \mathrm{~h}$ and $1.2 \mathrm{~h}$, respectively. Both were eastern events (Table 8.1).

Figure 8.9 shows that the $\mathrm{Ne} / \mathrm{O}$ ratios in these two events are similar and roughly constant ( $\sim 0.2)$ up to $\sim 30 \mathrm{MeV} /$ nucleon, but differ at higher energies with the ratio decreasing with energy in the 2013 Apr 11 event. On the other hand, the $(\mathrm{Fe}+\mathrm{Ni}) / \mathrm{O}$ ratios are quite strongly increasing with energy. The ratio is significantly higher in the event of 2013 Apr 11 than on 2012 Mar 7 reaching the maximum of $\sim 0.7$ in the 8-20 MeV/nucleon energy range and then decreasing with energy. The changes with energy in the abundance ratios and the differences between the two studied events can be explained by the differences in the energy where the high-energy spectral index is predominant. The high-energy spectral indices are believed to be dependent on the contribution of suprathermal flare material in the seed population. The high $(\mathrm{Fe}+\mathrm{Ni}) / \mathrm{O}$ ratio in the 2013 Apr 11 event indicates a more impulsive nature and larger flare material contribution in this smaller $\gamma$-ray event. It should be noted, however, that both of these events were large proton events lasting for several days. The abundance ratios can be compared with the average values of $\mathrm{Ne} / \mathrm{O}=$ 0.157 and $\mathrm{Fe} / \mathrm{O}=0.131$ in gradual solar energetic particle events at energies 2-15

Table 8.2 Heavy ion spectral parameters for the events of 2012 Mar 7 and 2013 Apr 11

\begin{tabular}{l|l|l|l|l|l|l}
\hline \multirow{3}{*}{ Event } & \multicolumn{3}{l}{ Oxygen } & \multicolumn{2}{l}{ Iron+Nickel } \\
\cline { 2 - 8 } & $\gamma_{\mathrm{a}}$ & $\gamma_{\mathrm{b}}$ & $E_{\mathrm{B}}(\mathrm{MeV})$ & $\gamma_{\mathrm{a}}$ & $\gamma_{\mathrm{b}}$ & $E_{\mathrm{B}}(\mathrm{MeV})$ \\
\hline 2012 Mar 7 & 1.03 & 5.22 & 9.8 & 0.3 & 4.9 & 6.4 \\
\hline 2013 Apr 11 & 1.53 & 4.01 & 18 & -0.2 & 3.1 & 3 \\
\hline
\end{tabular}





Fig. 8.9 Abundance ratios $\mathrm{Ne} / \mathrm{O}$ and $(\mathrm{Fe}+\mathrm{Ni}) / \mathrm{O}$ for the events of $2012 \mathrm{Mar} 7$ and $2013 \mathrm{Apr} 11$

$\mathrm{MeV} /$ nucleon (Reames 2014) and $\mathrm{Ne} / \mathrm{O}=0.478$ and $\mathrm{Fe} / \mathrm{O}=1.17$ on the average in impulsive SEP events (Reames et al. 2014).

\subsection{Summary and Discussion}

This overview on electromagnetic and SEP signatures that accompany sustained $\gamma$-ray emission of 25 events detected by Fermi/LAT is summarized as follows:

- High-energy $\gamma$-ray emission during the impulsive and early post-impulsive phase is accompanied by hard X-ray and radio signatures of energetic electron acceleration in the solar atmosphere. This is similar to the earlier findings for pion-decay $\gamma$-ray events (Kocharov et al. 1994; Trottet et al. 1994; Akimov et al. 1996) and GLEs (e.g., Klein et al. 2014). The manifestations in the early postimpulsive phase are visible at lower microwave frequencies than those of the impulsive phase, and at dm-to-m wavelengths (type IV bursts).

- $\quad \gamma$-ray emission lasting several hours is not accompanied by similarly extended emissions in hard X-rays or radio waves. In all cases (but 2011 Mar 7) these longduration events accompany the gradual decay of the soft X-ray flux. Although the parent soft X-ray burst starts sometimes more than an hour before the longlasting $\gamma$-ray enhancement, there are statistical relationships between (1) the importance of the parent soft X-ray burst and the peak flux of the long-duration $\gamma$-ray emission, and (2) the duration of the decay phase of the soft X-ray burst and the duration of the long-duration $\gamma$-ray emission.

- The 2011 Mar $7 \gamma$-ray event was accompanied by several distinct flares. But only the first of them showed conspicuous hard X-ray and radio emission. There is no indication that the long-lasting $\gamma$-ray emission could be understood as the superposition of several successive acceleration episodes in independent flares.

- Besides decaying soft X-ray emission and the related post-flare loop arcades, the only other electromagnetic counterpart of long-duration $\gamma$-rays are decametricto-hectometric type II bursts. They accompany the $\gamma$-ray emission of all longduration events. 
- SEP events are associated with all high-energy $\gamma$-ray events. In most wellconnected $\gamma$-ray events with long duration GOES/HEPAD detected protons above $300 \mathrm{MeV}$, and in several cases above $700 \mathrm{MeV}$. The $2011 \mathrm{Mar} 7 \gamma$-ray event is again an exception.

- At energies of several MeV to about $100 \mathrm{MeV}$ the SEP events are strong, but we found no characteristic feature that distinguishes them from strong SEP events in general. All are associated with DH type III bursts, as are SEP events in general (Cane et al. 2010; Vainio et al. 2013). When the solar release time of the first SEPs could be inferred from velocity dispersion analysis, it was found close to the interval of the type III bursts. A temporal relationship with the rise of the late long-duration $\gamma$-ray emission could not be excluded, but definite evidence for such a relationship could not be established either.

- The event-integrated abundances of $\mathrm{O}, \mathrm{Ne}, \mathrm{Fe}+\mathrm{Ni}$, and ${ }^{3} \mathrm{He}$ in the analysed SEP events show no unambiguous classification with respect to the conventional impulsive or gradual SEP values. There are, however, significant event-to-event variations in the ${ }^{3} \mathrm{He}$ and heavy ion abundance ratios and in their energy dependence. The sources of these variations and their possible relationships to the characteristics of the $\gamma$-ray emission need further analysis.

We conclude from the observations that pion-decay $\gamma$-rays during the impulsive and early post-impulsive phase are a manifestation of common particle acceleration with electrons, seen through hard X-ray and radio emissions. In the post-impulsive phase the signatures are mostly seen at long-centimetric to metric waves.

The association of $\gamma$-ray events with soft X-ray emission from the thermal plasma is not a direct clue to the origin of the mildly relativistic protons. Particle acceleration at the CME-driven shock in the high corona appears as an attractive interpretation, because it can explain that $\gamma$-rays are observed from flares behind the solar limb (Sect. 8.3) and because type II bursts are the unique systematic radio counterpart of long-duration $\gamma$-ray emissions (Sect. 8.4). Stereoscopic CME observations and sophisticated modelling allow one to estimate Mach numbers. Modelling of the shock acceleration (Chap. 9) shows that the highest Mach numbers, observed in restricted regions on the CME surface (Rouillard et al. 2016), may explain shock-accelerated protons up to $\mathrm{GeV}$ energies. This modelling shows furthermore that magnetic connections exist between the CME shock and the solar surface (Plotnikov et al. 2017). The interpretation faces two challenges from our present analysis: First, only a small fraction of the particles at the shock, less than $1 \%$ as estimated by a simple model, can reach the low solar atmosphere where nuclear interactions can take place. This may still be consistent with the estimation of proton numbers in space and the low solar atmosphere mentioned in Sect. 8.3. The second challenge is to explain why SEPs were only observed up to about $80 \mathrm{MeV}$ during the long-duration $\gamma$-ray event on 2011 Mar 7.

The HESPERIA sample of 25 events is too small to draw firm general conclusions on the origin of high-energy events and their relationship with SEPs. But it is rich enough to provide constraints on the processes of acceleration and coronal transport of mildly relativistic protons. 
Acknowledgements The authors are grateful to Gerald Share and Ron Murphy for preparing the text of Sect. 8.3.

\section{References}

Abdo, A.A., Ackermann, M., Ajello, M., et al.: Astrophys. J. 734, 116 (2011). doi:10.1088/0004$637 \mathrm{X} / 734 / 2 / 116$

Ackermann, M., Ajello, M., Allafort, A., et al.: Astrophys. J. 745, 144 (2012a). doi:10.1088/0004$637 \mathrm{X} / 745 / 2 / 144$

Ackermann, M., Ajello, M., Allafort, A., et al.: Astrophys. J. 748, 151 (2012b). doi:10.1088/0004$637 \mathrm{X} / 748 / 2 / 151$

Ackermann, M., Ajello, M., Albert, A., et al.: Astrophys. J. 787, 15 (2014). doi:10.1088/0004-63 $7 \mathrm{X} / 787 / 1 / 15$

Ackermann, M., Allafort, A., Baldini, L., et al.: Astrophys. J. 835, 219 (2017). doi:10.3847/1538-4 $357 / 835 / 2 / 219$

Ajello, M., Albert, A., Allafort, A., et al.: Astrophys. J. 789, 20 (2014). doi:10.1088/0004-63 7X/789/1/20

Akimov, V.V., Afanasyev, V.G., Belaousov, A.S., et al.: Sov. Astron. Lett. 18, 69 (1992)

Akimov, V.V., Ambrož, P., Belov, A.V., et al.: Sol. Phys. 166, 107 (1996)

Alexander, D., Dunphy, P.P., MacKinnon, A.L.: Sol. Phys. 151, 147 (1994). doi:10.1007/ BF00654088

Atwood, W.B., Abdo, A.A., Ackermann, M., et al.: Astrophys. J. 697, 1071 (2009). doi:10.1088/ 0004-637X/697/2/1071

Band, D., Matteson, J., Ford, L., et al.: Astrophys. J. 413, 281 (1993). doi:10.1086/172995

Bougeret, J.L., Kaiser, M.L., Kellogg, P.J., et al.: Space Sci. Rev. 71, 231 (1995). doi:10.1007/ BF00751331

Bougeret, J.L., Goetz, K., Kaiser, M.L., et al.: Space Sci. Rev. 136, 487 (2008). doi:10.1007/s11 214-007-9298-8

Cane, H.V., Richardson, I.G., von Rosenvinge, T.T.: J. Geophys. Res. (Space Phys.) 115, A08101 (2010). doi:10.1029/2009JA014848

Chupp, E.L., Ryan, J.M.: Res. Astron. 9, 11 (2009). doi:10.1088/1674-4527/9/1/003

Debrunner, H., Lockwood, J.A., Barat, C., et al.: Astrophys. J. 479, 997 (1997)

Dunphy, P.P., Chupp, E.L., Bertsch, D.L., et al.: Sol. Phys. 187, 45 (1999)

Forrest, D.J., Vestrand, W.T., Chupp, E.L., et al.: Int. Cosmic Ray Conf. 4, 146 (1985)

Forrest, D.J., Vestrand, W.T., Chupp, E.L., et al.: Adv. Space Res. 6(6), 115 (1986). doi:10.1016/02 73-1177(86)90127-4

Gold, R.E., Krimigis, S.M., Hawkins, S.E., et al.: Space Sci. Rev. 86, 541 (1998). doi:10.1023/A: 1005088115759

Huttunen-Heikinmaa, K., Valtonen, E., Laitinen, T.: Astron. Astrophys. 442, 673 (2005). doi:10.1051/0004-6361:20042620

Joshi, N.C., Uddin, W., Srivastava, A.K., et al.: 52, 1 (2013). doi:10.1016/j.asr.2013.03.009

Kanbach, G., Bertsch, D.L., Fichtel, C.E., et al.: Astron. Astrophys. Suppl. 97, 349 (1993)

Klein, K.L., Masson, S., Bouratzis, C., et al.: Astron. Astrophys. 572, A4 (2014). doi:10.1051/ 0004-6361/201423783

Kocharov, L.G., Kovaltsov, G.A., Kocharov, G.E., et al.: Sol. Phys. 150, 267 (1994)

Kocharov, L., Debrunner, H., Kovaltsov, G., et al.: Astron. Astrophys. 340, 257 (1998)

Kocharov, L., Laitinen, T., Vainio, R., et al.: Astrophys. J. 806, 80 (2015). doi:10.1088/0004637X/806/1/80

Kouloumvakos, A., Patsourakos, S., Nindos, A., et al.: Astrophys. J. 821, 31 (2016). doi:10.3847/ 0004-637X/821/1/31

Krucker, S., Larson, D.E., Lin, R.P., Thompson, B.J.: Astrophys. J. 519, 864 (1999) 
Kuznetsov, S.N., Kurt, V.G., Yushkov, B.Y., et al.: Sol. Phys. 268, 175 (2011). doi:10.1007/ s11207-010-9669-2

Kuznetsov, S.N., Kurt, V.G., Yushkov, B.Y., et al.: In: Kuznetsov V. (ed.) The Coronas-F Space Mission. Astrophysics and Space Science Library, vol. 400, p. 301. Springer, Berlin (2014). doi:10.1007/978-3-642-39268-9_10

Lario, D., Raouafi, N.E., Kwon, R.Y., et al.: Astrophys. J. 797, 8 (2014). doi:10.1088/0004$637 \mathrm{X} / 797 / 1 / 8$

Lario, D., Kwon, R.Y., Vourlidas, A., et al.: Astrophys. J. 819, 72 (2016). doi:10.3847/0004-6 37X/819/1/72

Leblanc, Y., Dulk, G.A., Bougeret, J.L.: In: Habbal, S.R., Esser, R., Hollweg, J.V., Isenberg, P.A. (eds.) Solar Wind Nine. American Institute of Physics Conference Series, vol. 471, pp. 83-86. AIP Press, New York (1999). doi:10.1063/1.58787

Lin, R.P., Dennis, B.R., Hurford, G.J., et al.: Sol. Phys. 210, 3 (2002). doi:10.1023/A:10224288 18870

Lockwood, J.A., Debrunner, H., Ryan, J.M.: Sol. Phys. 173, 151 (1997). doi:10.1023/A:1004908 209975

Malandraki, O.E., Agueda, N., Papaioannou, A., et al.: Sol. Phys. 281, 333 (2012). doi:10.1007/ s11207-012-0164-9

Mandzhavidze, N., Ramaty, R.: Astrophys. J. Lett. 396, L111 (1992). doi:10.1086/186529

Mewaldt, R.A., Cohen, C.M.S., Cook, W.R., et al.: Space Sci. Rev. 136, 285 (2008). doi:10.1007/ s11214-007-9288-X

Müller-Mellin, R., Böttcher, S., Falenski, J., et al.: Space Sci. Rev. 136, 363 (2008). doi:10.1007/ s11214-007-9204-4

Murphy, R.J., Dermer, C.D., Ramaty, R.: Astrophys. J. Supp. Ser. 63, 721 (1987). doi:10.1086/ 191180

Murphy, R.J., Share, G.H., Grove, J.E., et al.: Astrophys. J. 490, 883 (1997). doi:10.1086/304902

Nakajima, H., Sekiguchi, H., Sawa, M., et al.: Publ. Astron. Soc. Jpn. 37, 163 (1985)

Pesce-Rollins, M., Omodei, N., Petrosian, V., et al.: Astrophys. J. Lett. 805, L15 (2015). doi:10.1088/2041-8205/805/2/L15

Plotnikov, I., Rouillard, A.P., Share, G.H.: Astron. Astrophys. in press, (2017). doi:10.1051/0004-6361/201730804

Pulupa, M., Bale, S.D.: Astrophys. J. 676, 1330 (2008). doi:10.1086/526405

Rank, G., Ryan, J., Debrunner, H., et al.: Astron. Astrophys. 378, 1046 (2001). doi:10.1051/ 0004-6361:20011060

Reames, D.V.: Space Sci. Rev. 90, 413 (1999)

Reames, D.V.: Sol. Phys. 289, 977 (2014). doi:10.1007/s11207-013-0350-4

Reames, D.V., Cliver, E.W., Kahler, S.W.: Sol. Phys. 289, 3817 (2014). doi:10.1007/s11207014-0547-1

Reiner, M.J., Kaiser, M.L., Bougeret, J.L.: Astrophys. J. 663, 1369 (2007). doi:10.1086/ 510827

Rodríguez-Gasén, R., Kiener, J., Tatischeff, V., et al.: Sol. Phys. 289, 1625 (2014). doi:10.1007/ s11207-013-0418-1

von Rosenvinge, T.T., Reames, D.V., Baker, R., et al.: Space Sci. Rev. 136, 391 (2008). doi:10.10 07/s11214-007-9300-5

Rouillard, A.P., Plotnikov, I., Pinto, R.F., et al.: Astrophys. J. 833, 45 (2016). doi:10.3847/15384357/833/1/45

Ryan, J.M.: Space Sci. Rev. 93, 581 (2000)

Ryan, J.M., Lee, M.A.: Astrophys. J. 368, 316 (1991). doi:10.1086/169695

Ryan, J., Forrest, D., Lockwood, J., et al.: In: Ryan, J., Vestrand, W.T. (eds.) High-Energy Solar Phenomena - a New Era of Spacecraft Measurements. American Institute of Physics Conference Series, vol. 294, pp. 89-93 (1994). doi:10.1063/1.45205

Schlemm, C.E., Starr, R.D., Ho, G.C., et al.: Space Sci. Rev. 131, 393 (2007). doi:10.1007/ s11214-007-9248-5

Share, G.H., Murphy, R.J., Tolbert, K., et al.: Astrophys. J. (2017, Submitted)

Torsti, J., Valtonen, E., Lumme, M., et al.: Sol. Phys. 162, 505 (1995). doi:10.1007/BF00733438 
Torii, C., Tsukiji, Y., Kobayashi, S., et al.: Proceedings of the Research Institute of Atmospherics, Nagoya University, vol. 26, p. 129 (1979)

Trottet, G., Chupp, E.L., Marschhaeuser, H., Pick, M., Soru-Escaut, I., Rieger, E., Dunphy, P.P.: Astron. Astrophys. 288, 647 (1994)

Vainio, R., Valtonen, E., Heber, B., et al.: J. Space Weather Space Clim. 3, A12 (2013). doi:10.10 51/swsc/2013030

Vilmer, N., MacKinnon, A.L., Trottet, G., Barat, C.: Astron. Astrophys. 412, 865 (2003). doi:10.10 51/0004-6361:20031488

Vilmer, N., MacKinnon, A.L., Hurford, G.J.: Space Sci. Rev. 159, 167 (2011). doi:10.1007/s11 214-010-9728-x

Open Access This chapter is licensed under the terms of the Creative Commons Attribution 4.0 International License http://creativecommons.org/licenses/by/4.0/), which permits use, sharing, adaptation, distribution and reproduction in any medium or format, as long as you give appropriate credit to the original author(s) and the source, provide a link to the Creative Commons license and indicate if changes were made.

The images or other third party material in this chapter are included in the chapter's Creative Commons license, unless indicated otherwise in a credit line to the material. If material is not included in the chapter's Creative Commons license and your intended use is not permitted by statutory regulation or exceeds the permitted use, you will need to obtain permission directly from the copyright holder.

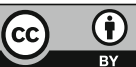

